\title{
Development and validation of a novel miRNA classifier as a prognostic signature for stage II/III colorectal cancer
}

\author{
Junlan Feng ${ }^{1}$, Qing Wei ${ }^{2}$, Muqing Yang ${ }^{1}$, Xiaodong Wang ${ }^{1}$, Bin Liu ${ }^{1}$ Jiyu Li ${ }^{1}$ \\ ${ }^{1}$ Department of General Surgery, Shanghai Tenth People's Hospital, School of Medicine, Tongji University, Shanghai, China; ${ }^{2}$ Department of \\ Pathology, Shanghai Tenth People's Hospital, School of Medicine, Tongji University, Shanghai, China \\ Contributions: (I) Conception and design: J Li, J Feng; (II) Administrative support: None; (III) Provision of study materials or patients: Q Wei; (IV) \\ Collection and assembly of data: J Feng, M Yang, X Wang, B Liu; (V) Data analysis and interpretation: J Feng, J Li; (VI) Manuscript writing: All \\ authors; (VII) Final approval of manuscript: All authors. \\ Correspondence to: Jiyu Li, MD, PhD. Department of General Surgery, Shanghai Tenth People's Hospital, School of Medicine, Tongji University, No. \\ 301 Middle Yan' Chang Road, Shanghai 200072, China. Email: lijiyu@tongji.edu.cn.
}

Background: The TNM staging remains the gold standard for determining the prognosis of patients with
colorectal cancer (CRC), which is inadequate at identifying the subset of high-risk stage II and III patients
that have a high potential of developing tumor recurrence and may experience death. Emerging evidence
indicates that not only microRNAs (miRNAs) play important functional role in CRC development but
may serve as important disease biomarkers. In this study we aimed to develop a miRNA-based classifier as a
prognostic signature for improving the clinical outcome of patients with stage II/III CRC. Methods: We performed a systematic and comprehensive discovery step to identify differentially expressed miRNAs in CRC. We subsequently determined the prognostic relevance of these miRNAs in stage II/III patients using qRT-PCR and developed a miRNA-based classifier for predicting disease-free survival (DFS) in a clinical cohort $(\mathrm{n}=186)$.

Results: Based upon miRNA expression profiling studies, we identified a panel of 10 miRNAs which are consistently differentially expressed in CRC vs. normal tissues. By using cox proportional hazard models, we then developed 6-miRNA-classifier (miR-183, -20a, -21, -195, -139 and -20a) to predict prognosis in clinical cohort, that had significantly superior predictive performance compared to other clinicopathological factors, and could successfully identify high-risk stage II and III CRC patients with poor prognosis [hazard ratio $(\mathrm{HR})=2.16 ; \mathrm{P}=0.0048]$. In a multivariate analysis, this miRNA-based classifier emerged as an independent prognostic signature for poor DFS.

Conclusions: Our miRNA-based classifier is a reliable predictive tool for determining prognosis in patents with stage II/III CRC, and might be able to identify high-risk patients that are candidates for more targeted personalized clinical management and surveillance.

Keywords: MicroRNA (miRNA); colorectal cancer (CRC); biomarker; prognosis

Submitted May 02, 2020. Accepted for publication Oct 19, 2020.

doi: $10.21037 /$ atm-20-1751

View this article at: http://dx.doi.org/10.21037/atm-20-1751

\section{Introduction}

Colorectal cancer (CRC) is currently the third most common cancer worldwide, with more than one million new cases diagnosed annually. The outcomes of CRC patients in early and late stages are drastically different, with the 5 -year survival rates of $\sim 93 \%$ for stage I disease and a dismal $8 \%$ for stage IV patients. Although $60 \%$ of CRC patients with (stages I-III) present with a resectable disease at the time of diagnosis, approximately $40-50 \%$ of such patients who undergo curative surgery or another $20-30 \%$ that are post-surgically treated with adjuvant chemotherapy, eventually relapse and experience a metastatic disease and 
eventual death (1-3). This clinical challenge highlights the limitation that the current golden standard of Tumor, Node, Metastasis (TNM)-based classification is inadequate at identifying the risk for tumor recurrence, which leads to potential under or over-treatment of a subset of patients with CRC.

At present, post-surgery, 5-fluorouracil (5FU)-based chemotherapy remains the standard of care treatment for some high-risk patients with stage II disease, and all patients with stage III CRC, as it helps improve survival rates by $10-20 \%(4,5)$. For stage II patients who present with specific high-risk clinical features, including advanced $\mathrm{T}$ stage, low differentiation grade, tumor perforation and few examined lymph nodes, are generally offered 5FUbased adjuvant treatment. Among these, $20 \%$ of stage II patients that are deemed low-risk clinically, experience tumor relapse. On the other hand, for stage III patients, $30-40 \%$ of patients do not show any evidence for tumor recurrence in 5 years even when left untreated, while $40 \%$ patients that receive adjuvant treatment still experience tumor recurrence and eventually die, highlighting the need for more intensive chemotherapy or the potential use of novel targeted therapies $(6,7)$. Taken together, these data underscore the need for identification of novel and robust prognostic biomarkers that can better guide treatment decisions in CRC patients with stage II and III disease.

Although in the recent years several studies have reported potential gene-expression based prognostic biomarkers for stage II/III patients, their adoption and routine use in the clinics have been hampered due to the need for high specimen quality and the lack of consensus and difficulties with analytical approaches. In this regard, microRNAs (miRNAs) have recently emerged as promising substrates for development of prognostic biomarkers in cancers, including CRC. MiRNAs are short (18-22 nt in length) and evolutionarily conserved non-coding RNAs. Compared to mRNAs or proteins, miRNAs are relatively immune to degradation by RNAses, and hence be readily detected and accurately quantified in a variety of clinical specimens including fresh frozen and formalin-fixed paraffinembedded (FFPE) tissues. Additionally, miRNAs have emerged as key frontiers in gene regulation due to their ability to regulate a broad range of biological processes in various human diseases, particularly cancer. We and others have previously highlighted that specific miRNAs may contribute to CRC pathogenesis, and many of these may serve as biomarkers for diagnosis, prognosis and metastasisprediction in CRC patients (8-11).
However, since the clinical usefulness of miRNAs in predicting the prognosis of stage II/III CRC patients remains unclear, we envisaged the present study to address this important gap in knowledge. Accordingly, we performed a systematic and comprehensive identification of CRC-specific miRNAs that are differentially expressed (DE) in stage II/III CRCs, followed by determining their combinatorial efficiency in predicting disease free survival by analyzing their expression in multiple, independent cohorts of patients with CRC.

We present the following article in accordance with the STROBE reporting checklist (available at http://dx.doi. org/10.21037/atm-20-1751).

\section{Methods}

\section{Patients and tumor specimens}

Clinical specimens analyzed for the miRNA classifier $(\mathrm{n}=186)$ were obtained from patients enrolled between 2008 and 2012 at the Shanghai Tenth People's Hospital, Shanghai, China. Patients were staged according to the American Joint Committee on Cancer (AJCC) staging guidelines. Detailed patient information is listed in Table 1. All CRC patients were followed up for survival for at least up to 5 years after surgery. Patients treated with radiotherapy or chemotherapy before surgery were excluded from this study. Personal or family history of polyposis or Lynch syndrome, personal history of inflammatory bowel disease, R1 or R2 resections (microscopic or macroscopic neoplastic involvement of surgical margins, respectively), and cases with lack of available FFPE tissues were also excluded from this study. The study was conducted in accordance with the Declaration of Helsinki (as revised in 2013) and was approved by the institutional review boards of Shanghai Tenth People's Hospital, School of Medicine, Tongji University (ID: KN84-01). Informed consent was taken from all the patients.

\section{Biomarker screening phase}

We performed an initial biomarker discovery phase wherein we performed an extensive published literature survey for all miRNA expression profiling studies published between 2006 and 2014. We included studies that exclusively performed direct comparison for miRNA expression profiles between normal and CRC tissues. MiRNAs were ranked according to several criteria as described previously (12): 
Table 1 Clinicopathological characteristics of patients in the clinical validation cohort

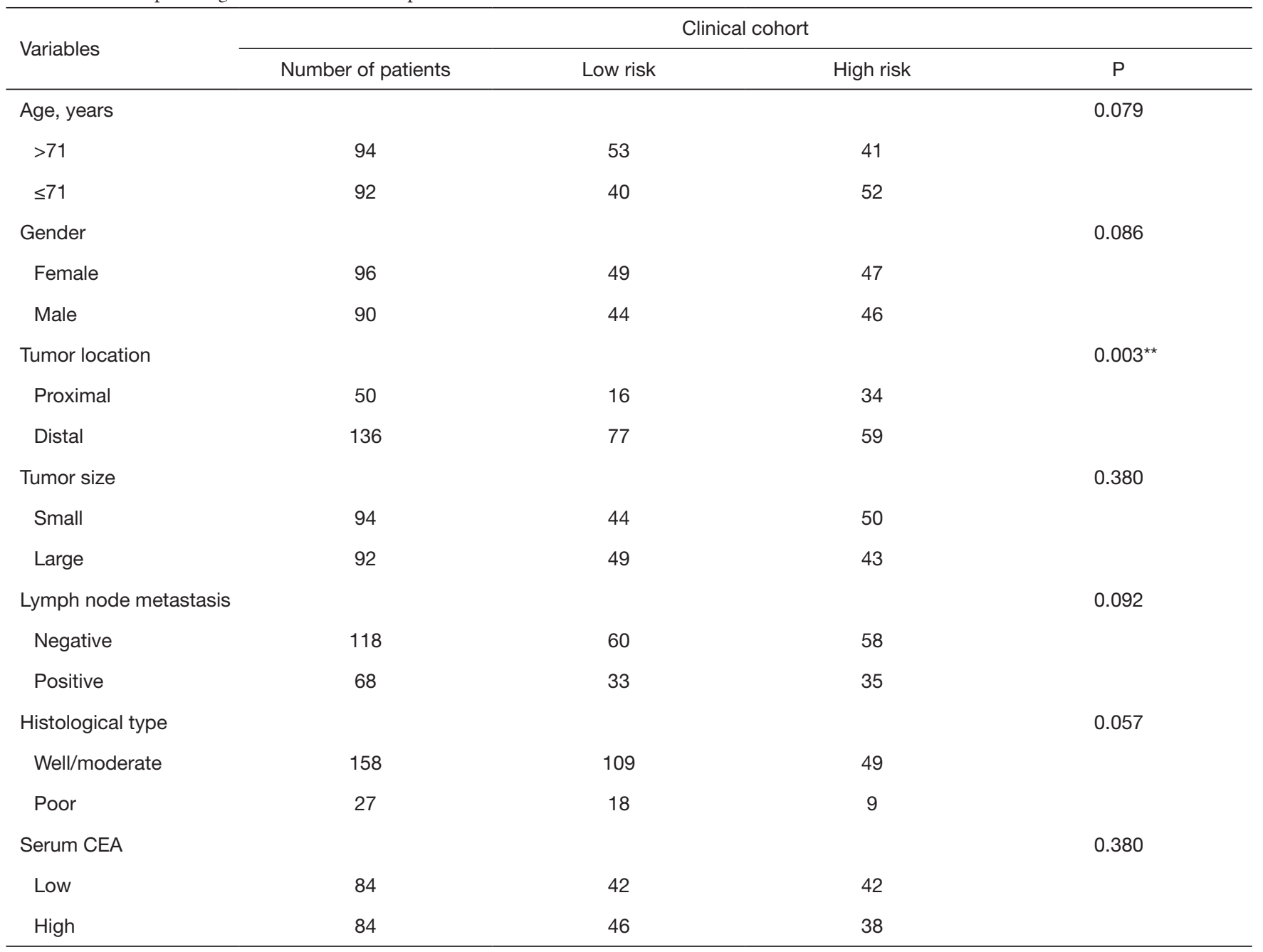

Pearson chi-squared testing was used to compare the correlation between triple-miRNA based classifier and clinical variables. ${ }^{* \star}, \mathrm{P}<0.01$. CEA, carcinoembryonic antigen.

(I) each miRNA was consistently reported as DE; (II) the direction of expression change (up- or down-regulation) was consistent across all studies; (III) the frequency of miRNA expression alteration was consistent and reported in multiple studies.

\section{Quantitative MiRNA expression analysis}

The miRNA expression analysis was performed using QuantStudio6 Flex Real-Time PCR System (Applied Biosystems, Foster City, CA, USA). All miRNA TaqMan probes were purchased from Ambion (Austin, TX, USA). The qRT-PCR assays were conducted using TaqMan MicroRNA Reverse Transcription Kit and TaqMan
Universal PCR Master Mix kit (Applied Biosystems, Foster City, CA, USA) according to manufacturer's instructions. The relative expression of miRNA was determined by $2^{-\Delta \Delta \mathrm{Ct}}$ method using miR-16 as a normalizer, as described previously (9,13-15).

\section{Statistical analysis}

All statistical analyses were performed using Medcalc version 12.3, SPSS version 13.0 or GraphPad Prism version 6.0. We conducted receiver operating characteristic (ROC) curves and calculated the area under the ROC curves (AUC) to evaluate the predictive power of candidate miRNAs for prognosticating CRC patients. For the disease-free 
survival (DFS) analysis, we defined the probability that patients remained free of tumor recurrence or death as the first event. Data were analyzed from the date of surgery to the time of the first event or the date on which data were censored, according to the Kaplan-Meier method, and the curves were compared using the log-rank test. To develop a miRNA panel and determining patient survival, we used Cox's proportional hazard regression models and obtained a risk score derived from this prediction model. We categorized patients into high-risk and low-risk group based on median cutoff value. Furthermore, we calculated estimate hazard ratios (HRs) for each miRNA, clinic-pathological variables and combination model, based on univariate and multivariate Cox proportional hazard regression models. All data were expressed as mean \pm standard deviation (SD). We compared two groups using the two-sided $\chi^{2}$ test for categorical variables. All $\mathrm{P}$ values were two-sided, and those less than 0.05 were considered statistically significant.

\section{Results}

\section{Systematic discovery and identification of potential miRNAs} for prognosis prediction in stage II and III CRC patients

During the past decade, several miRNAs with a prognostic potential for CRC patients have been identified, but majority of them have failed to validate across different studies. In order to avoid bias in selection of prognostic candidate miRNAs reported in previous studies, we initially performed a comprehensive and systematic literature review to identify most frequently and consistently reported miRNAs that are DE between CRC and normal tissues (Figure 1). Performing an exhaustive search of miRNA profiling studies, we identified a panel of 60 up-regulated and 41 down-regulated miRNAs that showed consistent data and were reported in at least 3 independent studies (Table S1). In order to narrow down this list further, we thereafter selected DE-miRNAs consistently reported in $\geq 10$ studies and gathered 10 miRNAs (miR-20a, -31, -183, -182, -21, -17,-145, -139, -195 and -215) which were significantly DE in CRCs, implicating their important role in the development of this disease, and their potential relevance in determining the clinical outcome of stage II/III CRC patients.

\section{Development of a prognostic miRNA classifier to predict survival in stage II/III patients}

We subsequently enrolled a clinical cohort of 186 patients to determine the optimal miRNA combinations for survival prediction. Accordingly, we measured expression level of each miRNA in CRC tissues, and the expression data was normalized by $Z$ score transformation, allowing the comparison of different cohort independent of the original signal intensities. Cox proportional hazard models were used to build a prognostic classifier. Of note, we adopted the back-step elimination algorithm to exclude non-significant confounders and thereafter identified miR-183, miR-21, miR20a, miR-139 and miR-195 was the optimal combination for survival prediction. We then derived a formula to calculate the risk score for their risk of disease recurrence for every patient based on their individual six miRNA expression levels, where the risk score $=(-1.2681 \times$ miR-139 $)+(-0.8916 \times$ miR-145 $)$ $+(0.7084 \times$ miR-183 $)+(1.4509 \times$ miR-195 $)+(0.9662 \times$ miR $20 \mathrm{a})+(-1.5493 \times \mathrm{miR}-21)$.

\section{Performance evaluation of the miRNA-classifier in a clinical cobort of stage II/III CRC patients}

We performed ROC analysis to evaluate the prediction accuracy of individual miRNAs and 6-miRNA classifier between DFS and recurrence/death. As shown in Table 2, our 6-miRNA-classifier (AUC: 0.705) significantly improved prediction ability of individual miRNA (AUC range, 0.530-0.643). Furthermore, compared to known clinicopathological risk factors, such as poor differentiation, lymph node metastasis, and tumor location, our newly developed miRNA-based classifier revealed the highest predictive accuracy (AUC $=0.715$; Figure $1 B$ ).

When we assessed the distribution of each patient's survival status and risk scores generated by this miRNAbased classifier, patients with lower risk scores showed better outcomes $v s$. those with higher risk scores (Figure 1C), highlighting its high predictive accuracy (Figure $2 A$ ). Based on cutoff value (the median value of all patients' risk scores), we divided patients into high-risk group and low-risk group and noted that high-risk group had worse prognosis compared to patients in the low-risk group (HR $=2.16, \mathrm{P}=0.0048$; Figure $2 B$ ). Furthermore, we observed high risk scores have strong tendency in association with proximal tumor $(\mathrm{P}=0.003)$, lymph node metastasis $(\mathrm{P}=0.092)$ and poor differentiation $(\mathrm{P}=0.057$, Table 1). In the univariate analysis, this 6-miRNA-classifier emerged as the strongest predictor of DFS (HR $=2.1604, \mathrm{P}=0.0059)$ compared to other clinicopathological variables such as serum CEA ( $\mathrm{HR}=1.8134, \mathrm{P}=0.0384)$, lymph node metastasis $(\mathrm{HR}$ $=1.5021, \mathrm{P}=0.1227)$ and tumor differentiation $(\mathrm{HR}=1.0408$, 
A

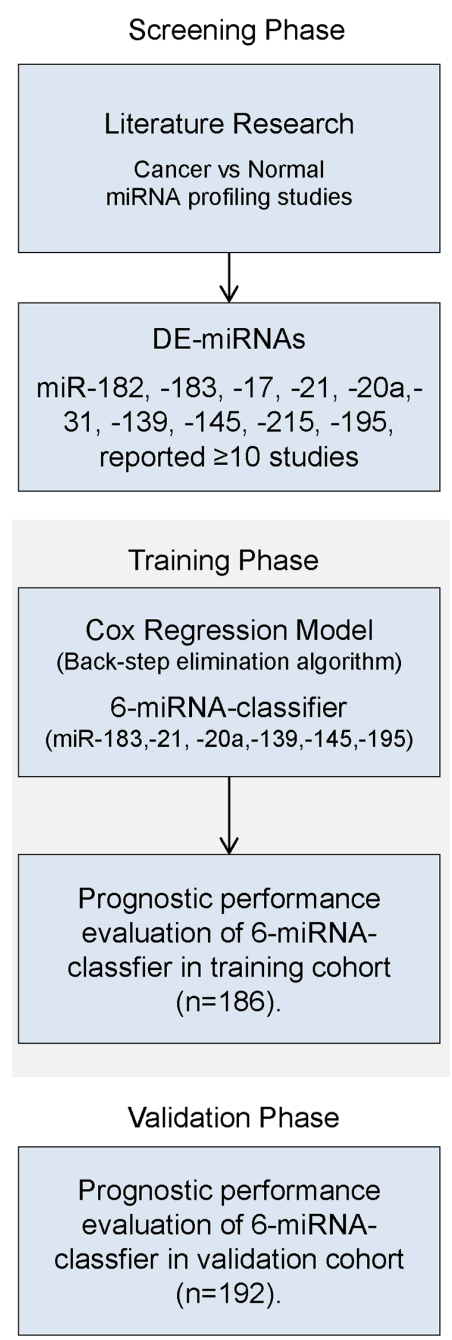

B

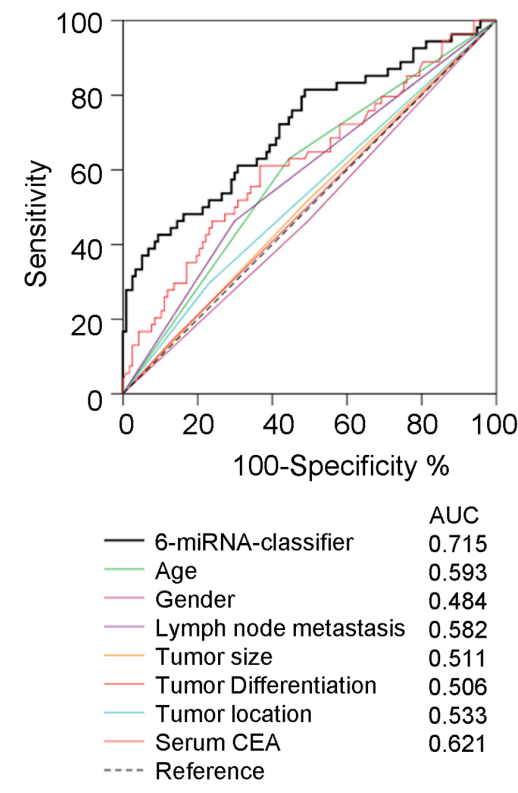

C

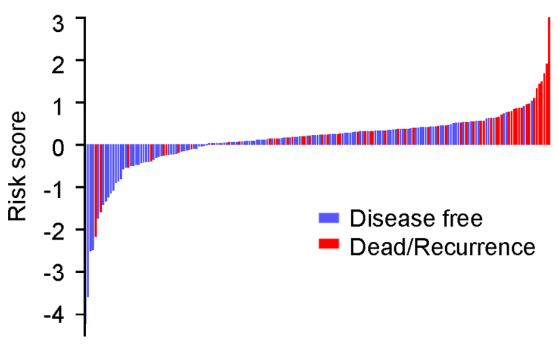

Figure 1 Overview of the study design and receiver operating characteristic (ROC) curves for the comparison of the prognostic accuracy of miRNA-classifier and clinicopathological variables in clinical cohort. (A) The overview of the study design. We performed a systematic and comprehensive discovery step to identify differentially expressed miRNAs in colorectal cancer (CRC). We subsequently determined the prognostic relevance of these miRNAs in stage II/III patients using qRT-PCR and developed a miRNA-based classifier for predicting disease-free survival (DFS) in a clinical cohort $(\mathrm{n}=186)$, which was later validated in an independent cohort $(\mathrm{n}=192)$. (B) The ROC analysis was used for the discrimination between disease free and recurrence or death cases. (C) The distribution of each patient's risk scores and survival status (recurrence or death). AUC, area under curve; DE, differentially expressed.

$\mathrm{P}=0$.9125). Consistently, multivariate analysis revealed that this 6-miRNA classifier was an independent prognostic factor in stage II/III CRC patients (HR $=2.5727, \mathrm{P}=0.0015$, Table 3).

When stratified by tumor stage, this miRNA-classifier still demonstrated clinically and statistically significant predictive power. As depicted in Figure 2B,C, the AUC of
miRNA-classifier is 0.73 and 0.67 in stage II and stage III respectively. In consistent, stage II patients with higher $v s$. lower risk scores had poor prognosis ( $\mathrm{HR}=2.09, \mathrm{P}=0.049$ ). When we analyzed the subset of stage III patients separately, the 6-miRNA-classifier also showed to be a highly predictive prognostic indicator, wherein patients in the high-risk group were more likely to have a poor outcome 
Table 2 The area under a ROC curve (AUC) of individual miRNAs and 6-miRNA classifier for disease free survival analysis in the clinical validation cohort

\begin{tabular}{llll}
\hline MiRNAs & & \multicolumn{2}{c}{ Clinical cohort } \\
\cline { 2 - 4 } miR-139 & AUC & SE & $95 \%$ CI \\
miR-145 & 0.643 & 0.0465 & $0.570-0.712$ \\
miR-183 & 0.585 & 0.0490 & $0.511-0.657$ \\
miR-195 & 0.625 & 0.0437 & $0.551-0.695$ \\
miR-20a & 0.575 & 0.0502 & $0.501-0.647$ \\
miR-21 & 0.626 & 0.0447 & $0.553-0.696$ \\
miR-17 & 0.530 & 0.0456 & $0.455-0.603$ \\
miR-182 & 0.608 & 0.0460 & $0.533-0.678$ \\
miR-215 & 0.633 & 0.0438 & $0.560-0.703$ \\
miR-31 & 0.542 & 0.0473 & $0.467-0.615$ \\
6-miRNA classifier & 0.559 & 0.0460 & $0.485-0.632$ \\
\hline
\end{tabular}

ROC, receiver operating characteristic; SE, standard error; $\mathrm{Cl}$, confidence interval.

vs. those with low-risk $(\mathrm{HR}=2.26, \mathrm{P}=0.041$; Figure $2 D)$. Notably, the high-risk stage II group yielded similar survival curves as stage III patients $(\mathrm{HR}=0.91, \mathrm{P}=0.7463$; Figure 2E), suggesting our classifier is able to identify high risk stage II group which has same prognosis as stage III group. Collectively, these results indicate that our newly developed 6-miRNA-classifier could successfully segregate high $v s$. low-risk patients with stage II/III disease. Which highlighting that our 6-miRNA based classifier is indeed a promising and reliable prognostic tool for identifying highrisk stage II and stage III patients, which has important implications for their clinical management.

\section{Discussion}

In this study, we have firstly performed a systematic discovery step, followed by development and validation of a novel prognostic tool based on a miRNA-classifier aimed at improving the predictive potential for the clinical outcomes of stage II/III CRC patients following surgery. Based upon a logical discovery, clinical validation steps, we provide data that our triple-miRNA based classifier was able to successfully discriminate high vs. low-risk CRC patients with a better predictive performance compared to the currently used TNM classification based clinicopathological variables used for determining therapeutic decision-making in stage II/III patients with CRC.
Although several studies have recently suggested that assessment of gene or protein expression changes may be used for prognostication of stage II/III CRC patients, methodological standardization including tissue handling, RNA or DNA processing, and lack of stability of these analytes have hampered the adoption of these biomarkers in routine clinical settings. In contrast, measurement of expression alterations of miRNAs offers several distinct advantages for their clinical use as biomarkers as these short non-coding RNA genes are highly stable in a variety of clinical specimens, have important functional role in regulating gene expression of key cancer-related genes, and their expression can be very accurately measured using simple PCR-based analytical tools. In view of these salient features of miRNAs, in this study we aimed to develop a miRNA-based predictive model for improved prognostication of stage II/III CRC patients.

In order to identify prognosis-related miRNAs for stage II/III CRC patients, we first selected robustly and DE miRNAs between cancer and normal tissues. We hypothesized that aberrant expression of these miRNAs may directly correlate with prognosis in stage II/III patients. Based upon a discovery step involving systematic literature review for miRNA expression profiling studies, we identified several DE miRNAs such as miR-145 (16-18), miR-21 $(19,20)$, miR-17 (21) and miR-20a (22). We subsequently measured expression level of 10 candidate miRNAs, which 
A

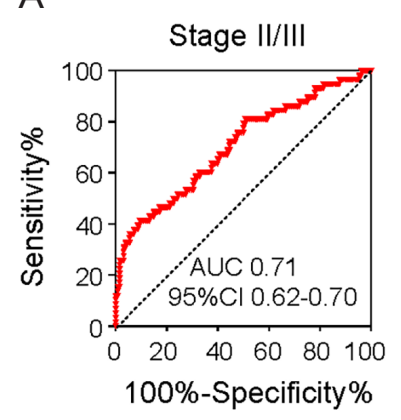

B

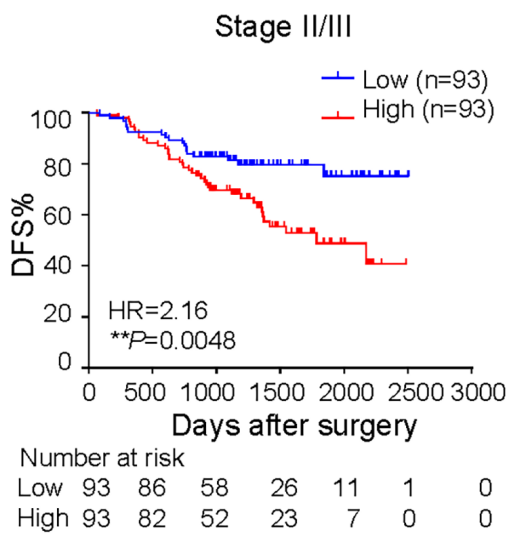

D

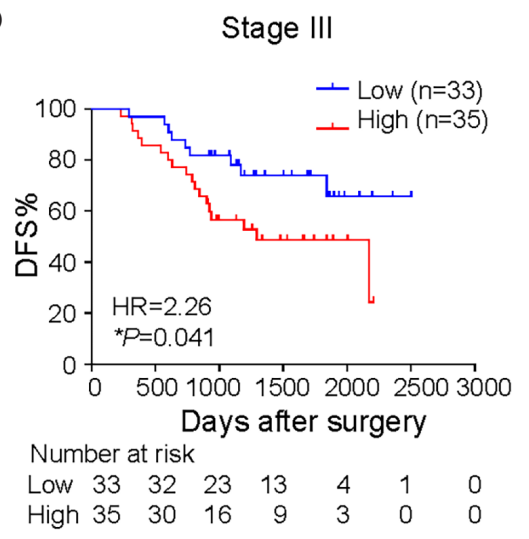

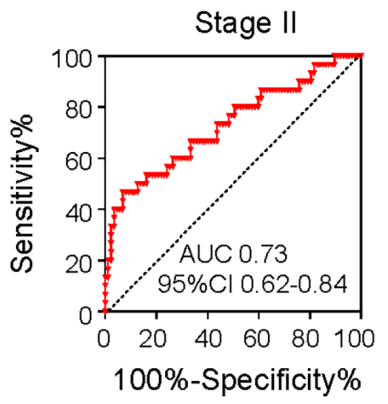

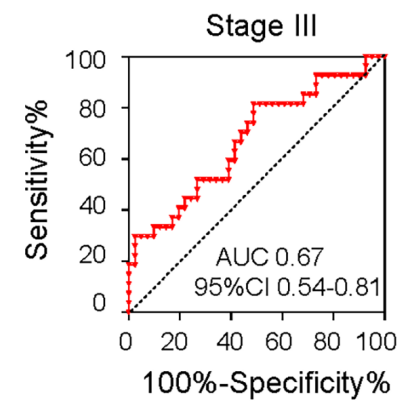

C

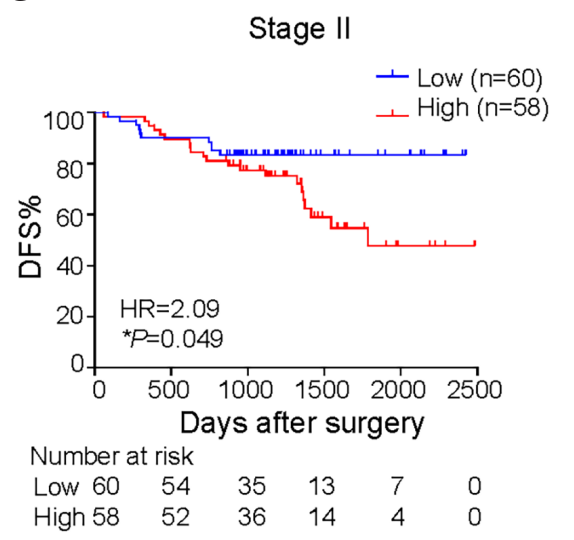

E

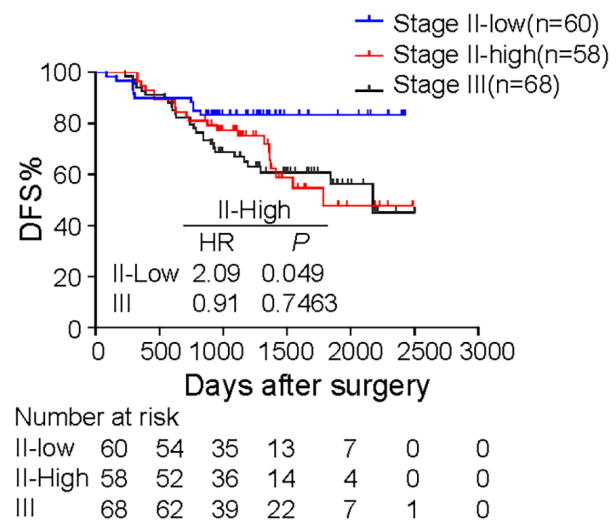

Figure 2 The prediction performance of 6-miRNA-classifier in the clinical cohort. (A) The predictive power of 6-miRNA-classifier was demonstrated in stage II/III patients by ROC analysis. (B,C,D,E) All the patients were divided into low and high-risk group based on risk scores calculated from 6-miRNA-classifier. The Kaplan-Meier analysis was used to estimate the prognosis of low and high-risk group in stage II and stage III. *, $\mathrm{P}<0.05$; **, $\mathrm{P}<0.01$. HR, hazard ratio; ROC, receiver operating characteristic; AUC, area under curve; DFS, diseasefree survival.

were reported to be differentially altered in colorectal $v s$. normal tissues at least 10 or more studies. We thereafter derived predictive models by using Cox's regression model, and identified a 6-miRNA-classifier consisting of miR183, miR-145, miR-20a, miR-21, miR-195 and miR-139, which was significantly superior in its prognostic accuracy 
Table 3 Univariate and multivariate association for the 6-miRNA-classifier and other clinicopathological characteristics with disease-free survival

\begin{tabular}{|c|c|c|c|c|c|c|}
\hline Clinical cohort & \multicolumn{3}{|c|}{ Univariate analysis } & \multicolumn{3}{|c|}{ Multivariate analysis } \\
\hline Age (>71 vs. $\leq 71$ years) & 1.6310 & $0.9636-2.7604$ & 0.0684 & - & - & - \\
\hline Gender (female vs. male) & 0.8628 & $0.5139-1.4484$ & 0.5765 & - & - & - \\
\hline Tumor location (proximal vs. distal) & 1.3471 & $0.7782-2.3316$ & 0.2872 & - & - & - \\
\hline Lymph node metastasis (pos vs. neg) & 1.5021 & $0.8961-2.5179$ & 0.1227 & - & - & - \\
\hline Differentiation (poor vs. well/mod) & 1.0408 & $0.5104-2.1224$ & 0.9125 & - & - & - \\
\hline Serum CEA (high vs. low) & 1.8134 & $1.0324-3.1853$ & $0.0384^{*}$ & 1.9074 & $1.0854-3.3519$ & $0.0248^{*}$ \\
\hline 6-miRNA-classifier (high vs. low) & 2.1604 & $1.2479-3.7401$ & $0.0059^{\star *}$ & 2.5727 & $1.4333-4.6179$ & $0.0015^{\star \star}$ \\
\hline
\end{tabular}

${ }^{*}, \mathrm{P}<0.05 ;{ }^{* *}, \mathrm{P}<0.01$. CEA, carcinoembryonic antigen; HR, hazard ratio; $\mathrm{Cl}$, confidence interval.

compared to the expression of individual miRNAs.

The biological function of these identified miRNAs selected for our classifier has been investigated previously. MiR-183 is a member of miR-183 cluster, which is comprised of miR-183, -182 and 96 . The miR-183 family is reported to be highly expressed in CRC and exerts its oncogenic activity via inhibition of several tumor suppressor genes (23). Furthermore, miR-183 was reported to be associated with poor prognosis in CRC patients (24). MiR195 , is a tumor suppressor, since its overexpression results in the inhibition of proliferation and metastasis in various cancers (25-28). MiR-195 is downregulated in CRC and its reduced expression associates with poor prognosis $(29,30)$. MiR-139 was shown to be down-regulated in a stagedependent manner in CRC, and regulates the expression of several oncogenes such as NOTCH1 (31), IGF1R $(32,33)$, MAPK, NF- $\mathrm{KB}$, and STAT3 (34). MiR-145 $(35,36)$ and miR-21 (37) are well-known miRNAs which function as tumor suppressor and onco-miR in CRC. MiR-20a was also reported to promote tumor development through suppression of GABBR1 (38). Considering the functional role as well as the clinical significance of these miRNAs in the development of cancer, it is rational to evaluate their expression in a miR-classifier for predicting prognosis of CRC patients.

With regards to potential limitations, our current study is retrospective in nature, and our results must be validated in future, prospective, multi-center clinical trials. In addition, some of the clinical parameters such as vascular invasion or number of analyzed lymph nodes were not consistently recorded or evaluated in our retrospective cohorts, which may be easier to address in a future well-defined patient cohort.

In conclusion, we provide compelling evidence that our newly developed miRNA-based prognostic classifier tool can effectively stratify patients with stage II/III CRCs into high and low risk groups based upon clinical outcomes, thereby adding significant prognostic value to the currently used clinicopathological risk factors used for such purposes. If validated in future studies, such a miRNA classifier potentially offers tremendous clinical value in directing personalized treatment regimens and clinical management of patients with stage II/III CRC.

\section{Acknowledgments}

We would like to express our deep and sincere gratitude to the patients and clinicians from the Department of General Surgery, Shanghai Tenth People's Hospital, School of Medicine, Tongji University, for their contributions to this study.

Funding: This work was supported by grants from the National Natural Science Foundation of China (grant No. 81470897 and No. 31741087).

\section{Footnote}

Reporting Checklist: The authors have completed the STROBE reporting checklist. Available at http://dx.doi. org/10.21037/atm-20-1751

Data Sharing Statement: Available at http://dx.doi. 
org/10.21037/atm-20-1751

Conflicts of Interest: All authors have completed the ICMJE uniform disclosure form (available at http://dx.doi. org/10.21037/atm-20-1751). The authors have no conflicts of interest to declare.

Ethical Statement: The authors are accountable for all aspects of the work in ensuring that questions related to the accuracy or integrity of any part of the work are appropriately investigated and resolved. The study was conducted in accordance with the Declaration of Helsinki (as revised in 2013) and was approved by the institutional review boards of Shanghai Tenth People's Hospital, School of Medicine, Tongji University (ID: KN84-01). Informed consent was taken from all the patients.

Open Access Statement: This is an Open Access article distributed in accordance with the Creative Commons Attribution-NonCommercial-NoDerivs 4.0 International License (CC BY-NC-ND 4.0), which permits the noncommercial replication and distribution of the article with the strict proviso that no changes or edits are made and the original work is properly cited (including links to both the formal publication through the relevant DOI and the license). See: https://creativecommons.org/licenses/by-nc-nd/4.0/.

\section{References}

1. Obrand DI, Gordon PH. Incidence and patterns of recurrence following curative resection for colorectal carcinoma. Dis Colon Rectum 1997;40:15-24.

2. O'Connell MJ, Campbell ME, Goldberg RM, et al. Survival following recurrence in stage II and III colon cancer: findings from the ACCENT data set. J Clin Oncol 2008;26:2336-41.

3. Andre T, Boni C, Navarro M, et al. Improved overall survival with oxaliplatin, fluorouracil, and leucovorin as adjuvant treatment in stage II or III colon cancer in the MOSAIC trial. J Clin Oncol 2009;27:3109-16.

4. Graham JS, Cassidy J. Adjuvant therapy in colon cancer. Expert Rev Anticancer Ther 2012;12:99-109.

5. Carethers JM. Systemic treatment of advanced colorectal cancer: tailoring therapy to the tumor. Therap Adv Gastroenterol 2008;1:33-42.

6. Andre T, Boni C, Mounedji-Boudiaf L, et al. Oxaliplatin, fluorouracil, and leucovorin as adjuvant treatment for colon cancer. N Engl J Med 2004;350:2343-51.
7. Kuebler JP, Wieand HS, O'Connell MJ, et al. Oxaliplatin combined with weekly bolus fluorouracil and leucovorin as surgical adjuvant chemotherapy for stage II and III colon cancer: results from NSABP C-07. J Clin Oncol 2007;25:2198-204.

8. Weng W, Feng J, Qin H, et al. An update on miRNAs as biological and clinical determinants in colorectal cancer: a bench-to-bedside approach. Future Oncol 2015;11:1791-808.

9. Hur K, Toiyama Y, Okugawa Y, et al. Circulating microRNA-203 predicts prognosis and metastasis in human colorectal cancer. Gut 2017;66:654-65.

10. Yamada A, Horimatsu T, Okugawa Y, et al. Serum miR-21, miR-29a, and miR-125b Are Promising Biomarkers for the Early Detection of Colorectal Neoplasia. Clin Cancer Res 2015;21:4234-42.

11. Hur K, Toiyama Y, Schetter AJ, et al. Identification of a metastasis-specific MicroRNA signature in human colorectal cancer. J Natl Cancer Inst 2015;107:dju492.

12. Ma Y, Zhang P, Yang J, et al. Candidate microRNA biomarkers in human colorectal cancer: systematic review profiling studies and experimental validation. Int J Cancer 2012;130:2077-87.

13. Okugawa $Y$, Toiyama $Y$, Toden $S$, et al. Clinical significance of SNORA42 as an oncogene and a prognostic biomarker in colorectal cancer. Gut 2017;66:107-17.

14. Han TS, Hur K, Xu G, et al. MicroRNA-29c mediates initiation of gastric carcinogenesis by directly targeting ITGB1. Gut 2015;64:203-14.

15. Hur K, Toiyama Y, Takahashi M, et al. MicroRNA-200c modulates epithelial-to-mesenchymal transition (EMT) in human colorectal cancer metastasis. Gut 2013;62:1315-26.

16. Su J, Liang H, Yao W, et al. MiR-143 and MiR-145 regulate IGF1R to suppress cell proliferation in colorectal cancer. PLoS One 2014;9:e114420.

17. Feng Y, Zhu J, Ou C, et al. MicroRNA-145 inhibits tumour growth and metastasis in colorectal cancer by targeting fascin-1. Br J Cancer 2014;110:2300-9.

18. Xu Q, Liu LZ, Qian X, et al. MiR-145 directly targets p70S6K1 in cancer cells to inhibit tumor growth and angiogenesis. Nucleic Acids Res 2012;40:761-74.

19. Peacock O, Lee AC, Cameron F, et al. Inflammation and MiR-21 pathways functionally interact to downregulate PDCD4 in colorectal cancer. PLoS One 2014;9:e110267.

20. Chang KH, Miller N, Kheirelseid EA, et al. MicroRNA-21 and PDCD4 expression in colorectal cancer. Eur J Surg Oncol 2011;37:597-603.

21. Ma Y, Zhang P, Wang F, et al. Elevated oncofoetal miR- 


\section{Page 10 of 10}

17-5p expression regulates colorectal cancer progression by repressing its target gene $\mathrm{P} 130$. Nat Commun 2012;3:1291.

22. Sokolova V, Fiorino A, Zoni E, et al. The Effects of miR20a on p21: Two Mechanisms Blocking Growth Arrest in TGF-beta-Responsive Colon Carcinoma. J Cell Physiol 2015;230:3105-14.

23. Sarver AL, French AJ, Borralho PM, et al. Human colon cancer profiles show differential microRNA expression depending on mismatch repair status and are characteristic of undifferentiated proliferative states. BMC Cancer 2009;9:401.

24. Zhou T, Zhang GJ, Zhou H, et al. Overexpression of microRNA-183 in human colorectal cancer and its clinical significance. Eur J Gastroenterol Hepatol 2014;26:229-33.

25. Wang M, Zhang J, Tong L, et al. miR-195 is a key negative regulator of hepatocellular carcinoma metastasis by targeting FGF2 and VEGFA. Int J Clin Exp Pathol 2015;8:14110-20.

26. Liu C, Guan H, Wang Y, et al. miR-195 Inhibits EMT by Targeting FGF2 in Prostate Cancer Cells. PLoS One 2015;10:e0144073.

27. Singh R, Yadav V, Kumar S, et al. MicroRNA-195 inhibits proliferation, invasion and metastasis in breast cancer cells by targeting FASN, HMGCR, ACACA and CYP27B1. Sci Rep 2015;5:17454.

28. Cai C, Chen QB, Han ZD, et al. miR-195 Inhibits Tumor Progression by Targeting RPS6KB1 in Human Prostate Cancer. Clin Cancer Res 2015;21:4922-34.

29. Wang X, Wang J, Ma H, et al. Downregulation of miR-195 correlates with lymph node metastasis and poor prognosis in colorectal cancer. Med Oncol 2012;29:919-27.

Cite this article as: Feng J, Wei Q, Yang M, Wang X, Liu B, Li J. Development and validation of a novel miRNA classifier as a prognostic signature for stage II/III colorectal cancer. Ann Transl Med 2021;9(9):747. doi: 10.21037/atm-20-1751

\section{Feng et al. miRNA-based classifier as a prognostic signature in CRC}

30. Wang L, Qian L, Li X, et al. MicroRNA-195 inhibits colorectal cancer cell proliferation, colony-formation and invasion through targeting CARMA3. Mol Med Rep 2014;10:473-8.

31. Zhang L, Dong Y, Zhu N, et al. microRNA-139-5p exerts tumor suppressor function by targeting NOTCH1 in colorectal cancer. Mol Cancer 2014;13:124.

32. Shen K, Mao R, Ma L, et al. Post-transcriptional regulation of the tumor suppressor miR-139-5p and a network of miR-139-5p-mediated mRNA interactions in colorectal cancer. FEBS J 2014;281:3609-24.

33. Shen K, Liang Q, Xu K, et al. MiR-139 inhibits invasion and metastasis of colorectal cancer by targeting the type I insulin-like growth factor receptor. Biochem Pharmacol 2012;84:320-30.

34. Zou F, Mao R, Yang L, et al. Targeted deletion of miR139-5p activates MAPK, NF- $\mathrm{BB}$ and STAT3 signaling and promotes intestinal inflammation and colorectal cancer. FEBS J 2016;283:1438-52.

35. Kent OA, McCall MN, Cornish TC, et al. Lessons from miR-143/145: the importance of cell-type localization of miRNAs. Nucleic Acids Res 2014;42:7528-38.

36. Luo X, Burwinkel B, Tao S, et al. MicroRNA signatures: novel biomarker for colorectal cancer? Cancer Epidemiol Biomarkers Prev 2011;20:1272-86.

37. Zhao J, Zhang Y, Zhao G. Emerging role of microRNA-21 in colorectal cancer. Cancer Biomark 2015;15:219-26.

38. Longqiu Y, Pengcheng L, Xuejie F, et al. A miRNAs panel promotes the proliferation and invasion of colorectal cancer cells by targeting GABBR1. Cancer Med 2016;5:2022-31. 
Supplementary

Table S1 Differential expressed miRNAs reported in at least 3 expression profiling studies (up: $\mathrm{n}=60$, down: $\mathrm{n}=41$ )

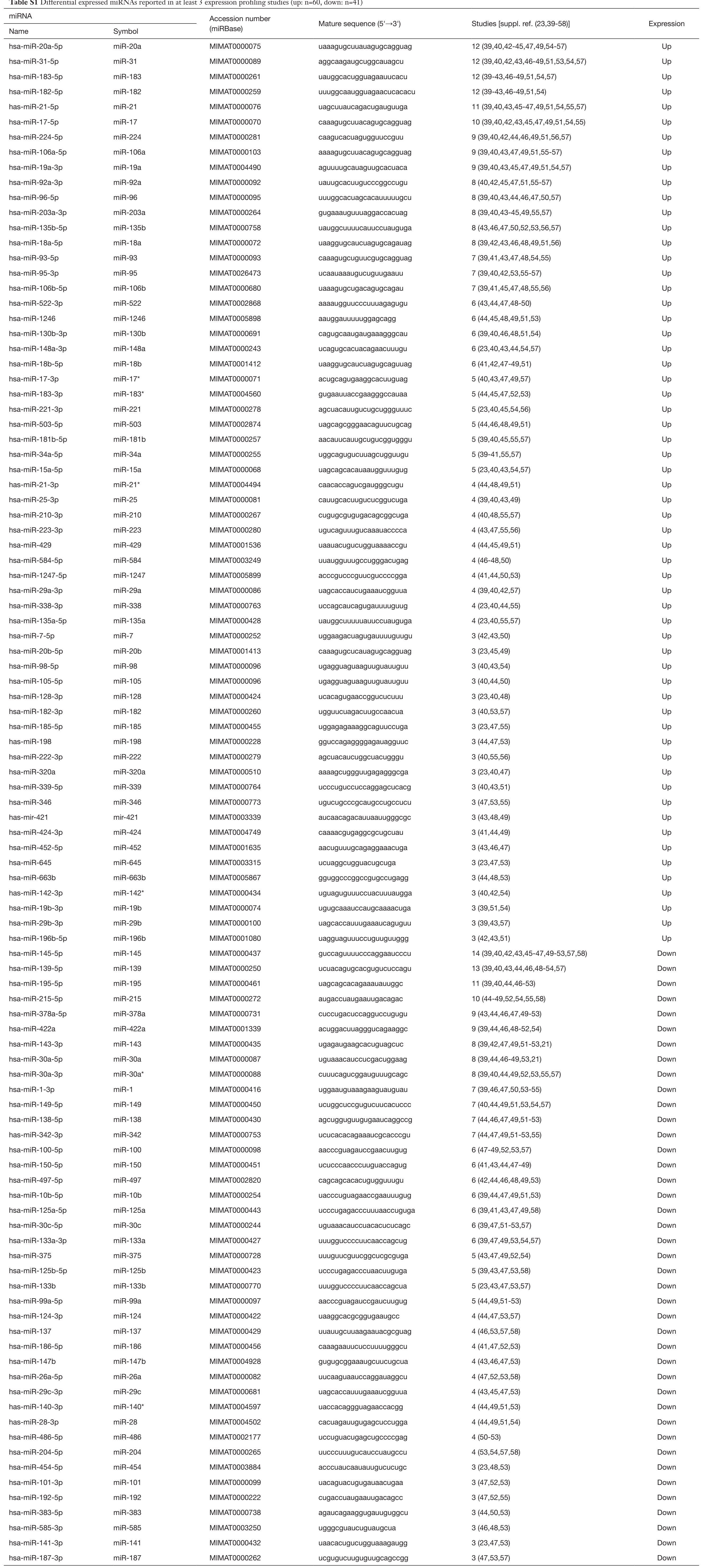




\section{References}

39. Arndt GM, Dossey L, Cullen LM, et al. Characterization of global microRNA expression reveals oncogenic potential of miR145 in metastatic colorectal cancer. BMC Cancer 2009;9:374.

40. Monzo M, Navarro A, Bandres E, et al. Overlapping expression of microRNAs in human embryonic colon and colorectal cancer. Cell Res 2008;18:823-33.

41. Ma Y, Zhang P, Wang F, et al. miR-150 as a potential biomarker associated with prognosis and therapeutic outcome in colorectal cancer. Gut 2012;61:1447-53.

42. Motoyama K, Inoue H, Takatsuno Y, et al. Over- and under-expressed microRNAs in human colorectal cancer. Int J Oncol 2009;34:1069-75.

43. Li X, Zhang G, Luo F, et al. Identification of aberrantly expressed miRNAs in rectal cancer. Oncol Rep 2012;28:77-84.

44. Yong FL, Law CW, Wang CW. Potentiality of a triple microRNA classifier: miR-193a-3p, miR-23a and miR-338-5p for early detection of colorectal cancer. BMC Cancer 2013;13:280.

45. Zhang JX, Song W, Chen ZH, et al. Prognostic and predictive value of a microRNA signature in stage II colon cancer: a microRNA expression analysis. Lancet Oncol 2013;14:1295-306.

46. Rohr C, Kerick M, Fischer A, et al. High-throughput miRNA and mRNA sequencing of paired colorectal normal, tumor and metastasis tissues and bioinformatic modeling of miRNA-1 therapeutic applications. PLoS One 2013;8:e67461.

47. Gaedcke J, Grade M, Camps J, et al. The rectal cancer microRNAome--microRNA expression in rectal cancer and matched normal mucosa. Clin Cancer Res 2012;18:4919-30.

48. Zhao Y, Miao G, Li Y, et al. MicroRNA- 130b suppresses migration and invasion of colorectal cancer cells through downregulation of integrin beta1 [corrected]. PLoS One 2014;9:e87938.

49. Pizzini S, Bisognin A, Mandruzzato S, et al. Impact of microRNAs on regulatory networks and pathways in human colorectal carcinogenesis and development of metastasis. BMC Genomics 2013;14:589.

50. Hamfjord J, Stangeland AM, Hughes T, et al. Differential expression of miRNAs in colorectal cancer: comparison of paired tumor tissue and adjacent normal mucosa using high-throughput sequencing. PLoS One 2012;7:e34150.

51. Piepoli A, Tavano F, Copetti M, et al. Mirna expression profiles identify drivers in colorectal and pancreatic cancers. PLoS One 2012;7:e33663.

52. Faltejskova P, Svoboda $M$, Srutova K, et al. Identification and functional screening of microRNAs highly deregulated in colorectal cancer. J Cell Mol Med 2012;16:2655-66.

53. Balaguer F, Moreira L, Lozano JJ, et al. Colorectal cancers with microsatellite instability display unique miRNA profiles. Clin Cancer Res 2011;17:6239-49.

54. Chang KH, Miller N, Kheirelseid EA, et al. MicroRNA signature analysis in colorectal cancer: identification of expression profiles in stage II tumors associated with aggressive disease. Int J Colorectal Dis 2011;26:1415-22.

55. Schetter AJ, Leung SY, Sohn JJ, et al. MicroRNA expression profiles associated with prognosis and therapeutic outcome in colon adenocarcinoma. JAMA 2008;299:425-36.

56. Ng EK, Chong WW, Jin H, et al. Differential expression of microRNAs in plasma of patients with colorectal cancer: a potential marker for colorectal cancer screening. Gut 2009;58:1375-81.

57. Bandres E, Cubedo E, Agirre X, et al. Identification by Real-time PCR of 13 mature microRNAs differentially expressed in colorectal cancer and non-tumoral tissues. Mol Cancer 2006;5:29.

58. Ng EK, Tsang WP, Ng SS, et al. MicroRNA-143 targets DNA methyltransferases 3A in colorectal cancer. Br J Cancer 2009;101:699-706. 